\title{
Computation of the Absorption Coefficient for Diatomic Molecules
}

\author{
Mats Larsson
}

Physics Department I, The Royal Institute of Technology, S-100 44 Stockholm, Sweden

\section{Introduction}

Molecules in stellar atmospheres play key roles, not only as promoters of our understanding of stars, but also as actors affecting the structures of the atmospheres. In particular cool star atmospheres are affected by molecular opacities, and during recent years it has become clear that also weak molecular lines are of crucial structural importance. Extensive molecular data are thus needed in order to correctly model the structure of a stellar atmosphere and the transport of radiation through it. The basis for how such molecular data are acquired constitutes the theme of the present article. As will follow below, results from different subfields of molecular physics are needed in order to obtain a complete picture of the absorption of radiation by molecules. This article will only consider allowed molecular electronic transitions, i.e. dipole transitions between different electronic states; however, the presentation and the formulae may easily be modified to be valid for transitions between levels within a single electronic state.

\section{Basic Concepts and Formulae}

Molecules are more complicated entities than atoms, and it could be useful to start this section with an introduction of the concept of a single rotational line in a molecular spectrum (Whiting et al. 1980). A rotational line is produced by the sum of all allowed transitions between degenerate magnetic substates, as illustrated in Fig. 1.

For some molecular electronic states there is a small splitting called $\Lambda$ doubling, originating from the interaction between the rotation of the nuclei and the electrons angular momentum, which can be difficult to resolve even by high resolution spectroscopy. Thus in some cases a rotational line has been defined as including both components of a $\Lambda$-doublet (Tatum 1967 and Schadee 1971). If one adopts the definition illustrated in Fig. 1, however, it follows that a $\Lambda$-doublet is composed of two rotational lines (Whiting \& Nicholls 1974). Figure 


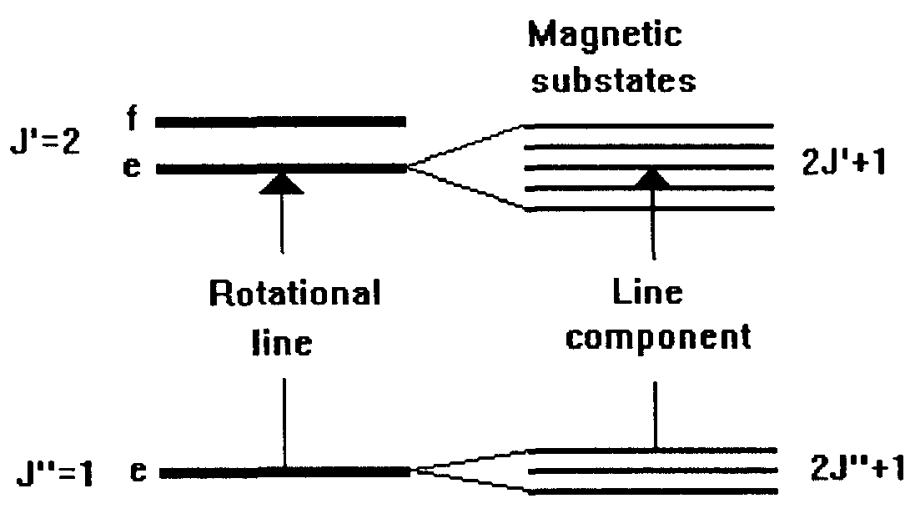

Fig. 1. A single molecular rotational line. The energy levels $J^{\prime}=2$ and $J^{\prime \prime}=1$ belong to two different electronic states. The upper electronic state is affected by $\Lambda$-doubling, which means that for each value of $J$ there is one level of $e$-type symmetry and one of $f$-type symmetry (see text). A single molecular rotational absorption line originates from a transition that terminats in one of the $A$-doublets $(J(e)=2-J(e)=1)$. A rotational level of the upper or lower electronic state, $J(e / f)$, is degenerate in the magnetic substates only. This is the origin of the $2 J+1$ degeneracy factor for a rotational level. The various line components of which a rotational line is composed can only be observed in the presence of a magnetic field (Zeeman effect).

2 further illustrates the point. It shows the energy level structures for ${ }^{2} \Pi$ and ${ }^{2} \Sigma^{+}$states and the first rotational line in each of the twelve different branches of a ${ }^{2} \Pi-{ }^{2} \Sigma^{+}$transition. The $A$-doubling occurs in the ${ }^{2} \Pi$ state and the levels are labelled according to their total parity, \pm , and their $e / f$ symmetry. The \pm and $e / f$ labels are two different bookkeeping devices for the same physical property, the $e / f$ labels being more convenient to use in connection with optical spectroscopy since they label a set of levels that usually can be described with a single energy level expression.

The absorption coefficient $K_{\nu}$ describes the attenuation of a beam of photons of frequency $\nu$ due to molecular absorption according to the expression

$$
I_{\nu}=I_{\nu}^{0} e^{-K_{\nu} \Delta x}
$$

where $I_{\nu}^{0}$ and $I_{\nu}$ are the intensities before and after transmission through a column of length $\Delta x$ of the molecular gas. For a transition $n \leftarrow m$ the integrated absorption coefficient can be expressed as

$$
\int K_{\nu} d \nu=\frac{\pi e^{2}}{m_{e} c} N_{m} f_{n m}
$$

where $N_{m}$ is the number density in the lower state $m$ of the molecule and $f_{n m}$ is the absorption oscillator strength (dimensionless). The labels $n$ and $m$ need to be specified if more useful formulas are to be developed. Let the upper state be specified by $n=\left|\Lambda^{\prime}, S^{\prime}, \Sigma^{\prime}, v^{\prime}, J^{\prime},(e / f)^{\prime}\right\rangle$ and the lower state by 


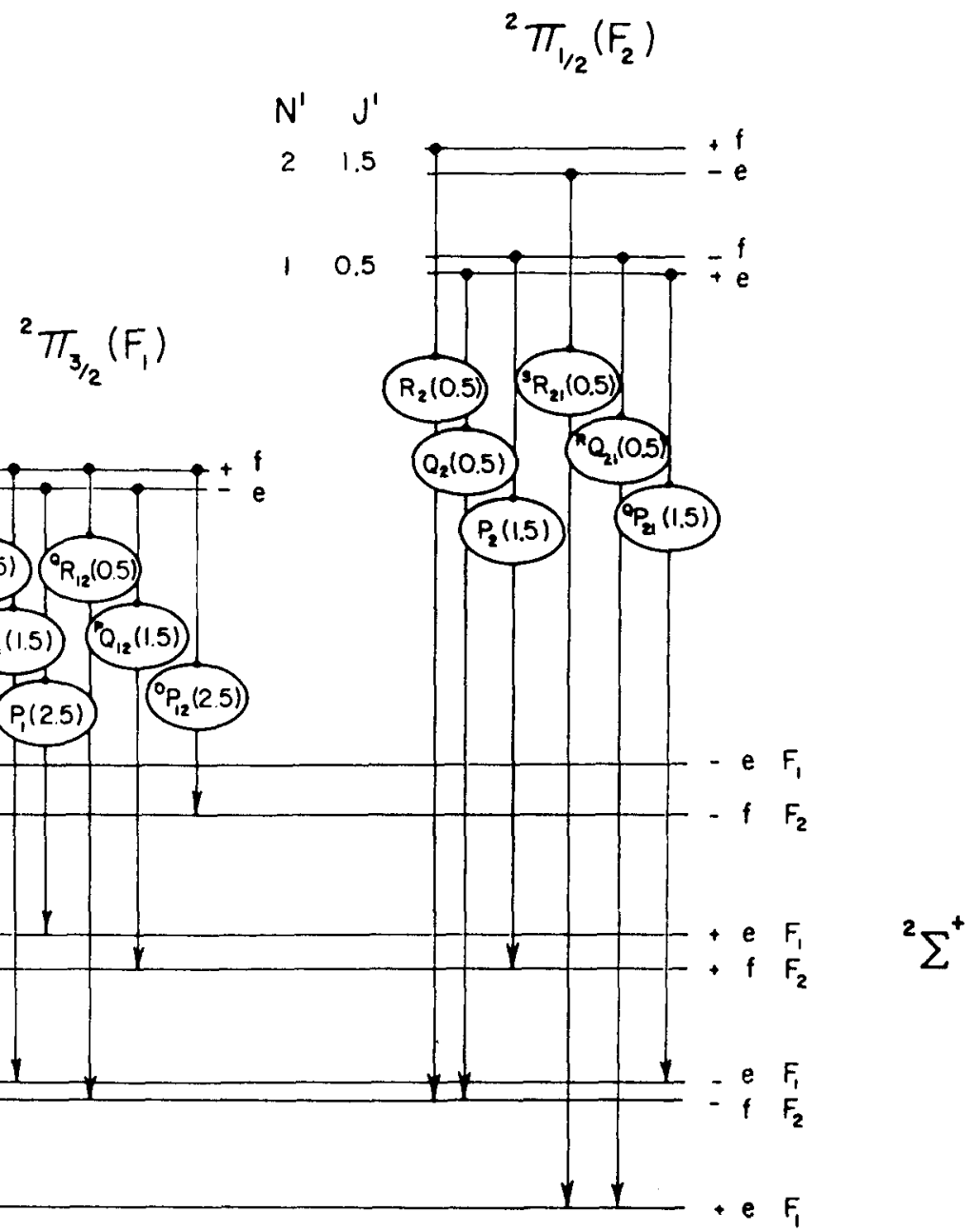

Fig. 2. Energy level diagram for $\mathrm{a}^{2} \Pi-^{2} \Sigma^{+}$transition. The lowest rotational line of each of the twelve different branches is shown. The $N$ quantum number is the total angular momentum of the molecule apart from the electron spin. Each filled circle in the upper state levels represents a transition from a single rotational level. (from Jørgensen \& Larsson 1990). 
$m=\left|\Lambda^{\prime \prime}, S^{\prime \prime}, \Sigma^{\prime \prime}, v^{\prime \prime}, J^{\prime \prime},(e / f)^{\prime \prime}\right\rangle$, where $\Lambda$ is the electronic state labelling (when the projection of the electrons angular momentum on the internuclear axis is $\Lambda=0,1$ or 2 this implies $\Sigma, \Pi$ or $\Delta$ states, respectively), $S$ is the total electron spin, $\Sigma$ is the specification of the spin multiplet ${ }^{1}, v$ is the vibrational quantum number, $J$ is the rotational quantum number and $(e / f)$ is the symmetry labelling.

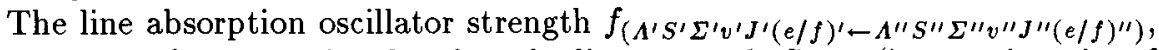
or, for short, $f_{v^{\prime} J^{\prime}, v^{\prime \prime} J^{\prime \prime}}$, is related to the line strength $S_{J^{\prime} J^{\prime \prime}}$ (in atomic units of $\left.e^{2} a_{0}^{2}\right)$ through

$$
f_{v^{\prime} J^{\prime}, v^{\prime \prime} J^{\prime \prime}}=\frac{8 \pi^{2} m_{e} c a_{0}}{3 h} \nu_{v^{\prime} J^{\prime}, v^{\prime \prime} J^{\prime \prime}} \frac{S_{J^{\prime} J^{\prime \prime}}}{2 J^{\prime \prime}+1}
$$

where $\nu_{v^{\prime} J^{\prime}, v^{\prime \prime} J^{\prime \prime}}$ denotes the energy difference between the upper and lower state expressed in wave numbers $\left(\mathrm{cm}^{-1}\right)$. In keeping with the tradition in molecular spectroscopy, the upper state is written first in the expression for the absorption oscillator strength although the lower state is the initial state in the absorption process. In order to avoid confusion an arrow $\leftarrow$ has been included in the full expression for $f$ to indicate the direction of the transition. The definition of the line strength, $S_{J^{\prime} J^{\prime \prime}}$, for a molecular transition was proposed by Whiting et al. (1980) and corresponds to Condon and Shortley's definition of the line strength for an atomic line (Condon \& Shortely 1935). It should be noted that $S_{J^{\prime} J^{\prime \prime}}$ is a shortened notation for $S_{\left(\Lambda^{\prime} S^{\prime} \Sigma^{\prime} v^{\prime} J^{\prime}(e / f)^{\prime} \rightarrow \Lambda^{\prime \prime} S^{\prime \prime} \Sigma^{\prime \prime} v^{\prime \prime} J^{\prime \prime}(e / f)^{\prime \prime}\right) \text {. The line }}$ strength is symmetric with respect to the direction of the transition, hence the two-directional arrow. The twelve rotational lines shown in Fig. 2, the first in each of the different branches in a ${ }^{2} \Pi-{ }^{2} \Sigma^{+}$transition, each has a separate value of $S_{J^{\prime} J^{\prime \prime}}$ and $f_{v^{\prime} J^{\prime}, v^{\prime \prime} J^{\prime \prime}}$.

It is in general a good approximation to separate the total wavefunction of a molecule into an electronic, a vibrational and a rotational part (the BornOppenheimer approximation). If this separation is carried through the line strength can be expressed as

$$
S_{J^{\prime} J^{\prime \prime}}=\left|\left\langle v_{J^{\prime}}^{\prime}\left|R_{e}(r)\right| v_{J^{\prime \prime}}^{\prime \prime}\right\rangle\right|^{2} \mathcal{S}_{J^{\prime} J^{\prime \prime}}
$$

Here $R_{e}(r)$ denotes the electronic transition moment (in atomic units of $e a_{0}$ ), $\mathcal{S}_{J^{\prime} J^{\prime \prime}}$ is the Hönl-London factor (dimensionless), and $\left|v_{J^{\prime}}^{\prime}\right\rangle$ and $\left|v_{J^{\prime \prime}}^{\prime \prime}\right\rangle$ are the vibrational wavefunctions for the upper and lower states; the index $J$ is used to indicate that the vibrational wavefunctions are obtained from the effective potential energy curve of the rotating system. Hence vibration-rotation interaction is taken into account. The concepts introduced in Eq. (4) will be discussed in more detail in the next section.

The transition rate per particle (transition probability) $A_{v^{\prime} J^{\prime}, v^{\prime \prime} J^{\prime \prime}}\left(\mathrm{s}^{-1}\right)$ for a spontaneous radiative transition $\left(\Lambda^{\prime} S^{\prime} \Sigma^{\prime} v^{\prime} J^{\prime}(e / f)^{\prime}\right) \rightarrow\left(\Lambda^{\prime \prime} S^{\prime \prime} \Sigma^{\prime \prime} v^{\prime \prime} J^{\prime \prime}(e / f)^{\prime \prime}\right)$ is related to the electronic transition moment and Hönl-London factor through

$$
A_{v^{\prime} J^{\prime}, v^{\prime \prime} J^{\prime \prime}}=\frac{64 \pi^{4} e^{2} a_{0}^{2}}{3 h} \nu_{v^{\prime} J^{\prime}, v^{\prime \prime} J^{\prime \prime}}^{3} \frac{\left|\left\langle v_{J^{\prime}}^{\prime}\left|R_{e}(r)\right| v_{J^{\prime \prime}}^{\prime \prime}\right\rangle\right|^{2} \mathcal{S}_{J^{\prime} J^{\prime \prime}}}{\left(2 J^{\prime}+1\right)}
$$

${ }^{1}$ This should not be confused with a $\Sigma$ state, which implies that $A=0$; in Fig. 2 the ${ }^{2} \Pi_{3 / 2}$ state is obtained for $\Sigma=1 / 2$ and ${ }^{2} \Pi_{1 / 2}$ for $\Sigma=-1 / 2$. 
Relations between various variables describing the radiative properties of diatomic molecules can be found in the paper by (Larsson 1983).

It should finally be noted that it is very common to use the concept of band absorption oscillator strength and to discuss transition probabilities in terms of an electronic-vibrational transition $\left(\Lambda^{\prime} v^{\prime}\right) \rightarrow\left(\Lambda^{\prime \prime} v^{\prime \prime}\right)$. In these cases $f_{v^{\prime} v^{\prime \prime}}$ and $A_{v^{\prime} v^{\prime \prime}}$ are obtained as an averaged sum (Larsson 1983).

\section{Calculations of absorption oscillator strength}

From an astrophysicists point of view the oscillator strength is often the desired radiative property to be used for describing absorption of radiation by molecules. In fact, it is even more appropriate to say that it is the $g f$-value, i.e. the absorption oscillator strength multiplied by the degeneracy factor for the lower state. For the line absorption oscillator strength this factor is always $\left(2 J^{\prime \prime}+1\right)$, while if the band absorption oscillator strength is used, the degenary factor depends on the electronic transition (Larsson 1983). In the line data tape for the CN molecule, which will be described below, the $g f$-value is always $\left(2 J^{\prime \prime}+1\right) f_{v^{\prime} J^{\prime}, v^{\prime \prime} J^{\prime \prime}}$.

The separation of the molecular wavefunction into three parts, as expressed in Eq. (4), makes it natural to divide the discussion of how to calculate $g f$-values into three parts. In the first part we will discuss how to obtain the electronic transition moment, in the second part how to calculate vibrational wavefunctions, and in the third part how to obtain Hönl-London factors.

The definition of the electronic transition moment for a molecular transition is intimately connected to the exact way the electronic and rotational parts of the total wavefunction are separated. We need not go into the details here; it suffices to note that, according to the recommendations by Whiting et al. (1980), the electronic transition moment for a parallel transition $\left(\Lambda^{\prime}=\Lambda^{\prime \prime}\right)$ should be expressed as

$$
R_{e}=\left\langle\Lambda^{\prime} S^{\prime} \Sigma^{\prime}\left|\mu_{z}\right| \Lambda^{\prime \prime} S^{\prime \prime} \Sigma^{\prime \prime}\right\rangle
$$

and for a perpendicular transition $\left(\Lambda^{\prime}=\Lambda^{\prime \prime} \pm 1\right)$

$$
R_{e}=\left\langle\Lambda^{\prime} S^{\prime} \Sigma^{\prime}\left|\frac{1}{\sqrt{2}}\left(\mu_{x} \pm i \mu_{y}\right)\right| \Lambda^{\prime \prime} S^{\prime \prime} \Sigma^{\prime \prime}\right\rangle
$$

where $\mu_{z}$ and $\frac{1}{\sqrt{2}}\left(\mu_{x} \pm i \mu_{y}\right)$ are different components of the electronic transition moment operator. These expressions are valid for spin-allowed transitions, which implies that $S^{\prime}=S^{\prime \prime}$.

Prior to the development of modern ab initio quantum chemistry methods, the electronic transition moment could only be obtained by means of rather hazardous inversions of experimental data obtained from intensity measurements or measurements of fluorescence lifetimes. Nowadays, with the level of accuracy that can be obtained with computer based state-of-the-art quantum chemistry methods (see Langhoff \& Bauschlicher, Malmqvist, and Peyerimhoff, this volume), the fastest and most accurate way to obtain $R_{e}(r)$ is to calculate it from $a b$ 
initio molecular electronic wavefunctions. The procedure is to calculate the electronic wavefunctions for the upper $\left(\left|\Lambda^{\prime} S^{\prime} \Sigma^{\prime}\right\rangle\right)$ and lower $\left(\left|\Lambda^{\prime \prime} S^{\prime \prime} \Sigma^{\prime \prime}\right\rangle\right)$ electronic states for a number of different values of the internuclear separation. For a diatomic molecule it is in general sufficient to carry out these calculations at 10-20 different internuclear distances. The energy values obtained in such calculations constitute a set of data which, by interpolation, provide approximate potential curves for the two states. The electronic wavefunctions can then be used to evaluate the matrix elements given in Eqs. (6) and (7), and the electronic transition moment function, $R_{e}(r)$ is obtained by interpolation between the 10-20 internuclear distances used in the calculation. It should finally be pointed out that if $a b$ initio calculated electronic transition moments are used one should make sure that they are defined in accordance with Eqs. (6) and (7) (for a brief discussion of this issue, see Whiting et al. (1980) and Larsson (1983)).

Once the electronic transition moment function has been obtained, the question is how to assess its accuracy. The method of choice is to compute the radiative lifetime of the upper state and compare these results with available experimental results. This is readily done by summation of $A_{v^{\prime} J^{\prime}, v^{\prime \prime} J^{\prime \prime}}$ in Eq. (5) over all lower states $v^{\prime \prime} J^{\prime \prime}$. Ideally, experimental results obtained with different techniques agree, and the comparison of the theoretical results with experiment provides a direct assessment of the theoretical method. For some molecules this is the case, as for example CH (Larsson \& Siegbahn 1983). In other cases, if carefully designed, $a b$ initio calculations can even be used to assess different experimental techniques (Bauschlicher \& Langhoff 1987). Unfortunately there are also molecules for which there seems to be a never ending confusion both on the experimental and theoretical side. As we shall see below, $\mathrm{CN}\left(\mathrm{A}^{2} \Pi-\mathrm{X}^{2} \Sigma^{+}\right)$is one of these molecules.

When the electronic transition moment function, $R_{e}(r)$, has been obtained, the next step is to calculate the matrix elements $\left\langle v_{J^{\prime}}^{\prime}\left|R_{e}(r)\right| v_{J^{\prime \prime}}^{\prime \prime}\right\rangle$, as follows from Eqs. (3) and (4). The vibrational wavefunctions $\left|v_{J}\right\rangle$ are obtained as solutions to the Schrödinger equation describing the oscillatory motion of the two nuclei in a potential $V_{0}(r)$ for the non-rotating molecule (if one should be a purist, it is the wavefunctions for $\left|v_{J^{\prime}=A^{\prime}}^{\prime}\right\rangle$ and $\left|v_{J^{\prime \prime}=A^{\prime \prime}}^{\prime \prime}\right\rangle$ which are obtained). Provided that potential curves are available for the two electronic states, the calculation of wavefunctions $\left|v_{J}\right\rangle$ is straightforward and demands orders of magnitude less computer time as compared to $a b$ inito calculations of the electronic wavefunctions. There are several methods that can be used to obtain potential curves. We have already mentioned that $a b$ initio calculation is one such method, and in fact the only available route if there are no experimental data to resort to. However, if results from high resolution molecular spectroscopy are available, it is better to use these data together with an inversion procedure to obtain the potential curve. The Rydberg-Klein-Rees (RKR) procedure is the most widely used method among molecular spectroscopists for deriving $V_{0}(r)$. The method uses energy levels and molecular constants derived from molecular spectroscopy and, via a semiclassical quantization condition, generates pairs of turning points which define a potential curve (Lefebvre-Brion \& Field 1986). There are also 
slightly modified versions of the RKR procedure, for example the Klein-Dunham method (Jarmain 1971), identical to RKR except for very light molecules. It is rare that experimental data allows the construction of an RKR potential all the way to the dissociation limit. In practise one has to make an extrapolation of the potential outside the range of internuclear distances covered by the RKR turning points. One way to do this is to extend the curve with a Morse potential, another way is to make an extension with an ab initio potential.

When the potential energy curves have been constructed, the vibrational wavefunctions $\left|v_{J^{\prime}}^{\prime}\right\rangle$ and $\left|v_{J^{\prime \prime}}^{\prime \prime}\right\rangle$ are calculated by numerical integration of the Schrödinger equation. Normally the computer programme used to obtain the potential $V_{0}(r)$ (RKR or Klein-Dunham methods) also provides the necessary routines to obtain $\left|v_{J}\right\rangle$. The potential $V_{0}(r)$ does not include the rotational motion of the nuclei (hence the index 0 in $V_{0}(r)$ ). When a molecule rotates, an interaction between the vibrational and rotational motion is induced. This interaction can be accounted for in calculations of line absorption oscillator strengths by adding a term $\left(h / 8 \pi^{2} \mu r^{2}\right) J(J+1)$ to $V_{0}(r)^{2}$. In the calculation of the line data tape for the CN molecule (Jørgensen \& Larsson 1990), the $J(J+1)$-term was always added to $V_{0}(r)$ and the calculation of vibrational wavefunctions was carried out separately for each value of $J$. The computer programme TRAPRB (Jarmain 1971, Jarmain \& McCallum 1971) was used to obtain the $\left|v_{J}\right\rangle$ wavefunctions.

The calculation of the matrix elements $\left\langle v_{J^{\prime}}^{\prime}\left|R_{e}(r)\right| v_{J^{\prime \prime}}^{\prime \prime}\right\rangle$ is performed using the molecule-fixed components of the electronic transition moment operator (see Eqs. (6) and (7)), i.e. a coordinate system attached to the rotating molecule. Observations of molecular line intensities, however, are performed in a laboratoryfixed coordinate system. Hence a transformation of coordinate system is needed, and it is this transformation which introduces the Hönl-London factors (Hougen 1970 ), or rotational line intensity factors as they are also called. The HönlLondon factors enter the expression for the line strength according to Eq.(4). Originally the Hönl-London factors were calculated analytically (Kovacs 1969) and involved approximations to make the calculations tractable. More recently computer codes have been developed which calculate the Hönl-London factors numerically by means of suitable transformations (Whiting 1972, Whiting 1973, Whiting \& Nicholls 1974). The theory for Hönl-London factors has also been formulated in a comprehensive way by Veseth (1986) using group theory.

If the Hönl-London factors are summed over all transitions from the group of $\left(2-\delta_{0, A^{\prime \prime}}\right)\left(2 S^{\prime \prime}+1\right)$ levels ${ }^{3}$ with a specific $J^{\prime \prime}$ value to the $\left(2-\delta_{0, A^{\prime}}\right)\left(2 S^{\prime}+1\right)$ levels with $J^{\prime}=J^{\prime \prime}$ and $J^{\prime}=J^{\prime \prime} \pm 1$, one obtains $\left(2-\delta_{0, A^{\prime}} \delta_{0, A^{\prime \prime}}\right)\left(2 S^{\prime \prime}+1\right)\left(2 J^{\prime \prime}+1\right)$ (Whiting \& Nicholls 1974). If, for example, the Hönl-London factor for each branch in the ${ }^{2} \Pi-{ }^{2} \Sigma^{+}$transition in Fig.2 is summed for a specific value of $J^{\prime \prime}$ one obtains

$$
\Sigma \mathcal{S}=\mathcal{S}\left(R_{1}\right)+\mathcal{S}\left(Q_{1}\right)+\mathcal{S}\left(P_{1}\right)+\mathcal{S}\left({ }^{Q} R_{12}\right)+\mathcal{S}\left({ }^{P} Q_{12}\right)+\mathcal{S}\left({ }^{o} P_{12}\right)
$$

${ }^{2} \mu$ is the reduced mass of the molecule and should not be confused with the notation for the transition moment operator in Eqs. (6) and (7).

${ }^{3} \delta_{0, A^{\prime \prime}}$ is a Kronecker delta, i.e. $\delta_{0, A^{\prime \prime}}=1$ for $A^{\prime \prime}=0$ and $\delta_{0, A^{\prime \prime}}=0$ for $\Lambda^{\prime \prime} \neq 0$. 


$$
\begin{aligned}
& +\mathcal{S}\left(R_{2}\right)+\mathcal{S}\left(Q_{2}\right)+\mathcal{S}\left(P_{2}\right)+\mathcal{S}\left({ }^{S} R_{21}\right)+\mathcal{S}\left({ }^{R} Q_{21}\right)+\mathcal{S}\left({ }^{Q} P_{21}\right) \\
= & \left(2-\delta_{0, \Lambda^{\prime}} \delta_{0, \Lambda^{\prime \prime}}\right)\left(2 S^{\prime \prime}+1\right)\left(2 J^{\prime \prime}+1\right)=4\left(2 J^{\prime \prime}+1\right)
\end{aligned}
$$

The sum rule is symmetrical in $J^{\prime}$ and $J^{\prime \prime}$ which means that the " notation on $J$ is redundant. This completes the calculation of $f_{v^{\prime} J^{\prime}, v^{\prime \prime} J^{\prime \prime}}$ according to Eqs. (3) and (4).

\section{Molecular absorption coefficients}

It follows from Eq. (2) that, in order to calculate the absorption coefficient for a molecular transition, one needs, in addition to the oscillator strength, also the number density in the lower state $\left(N_{m}\right.$ in Eq. (2)). In a molecule the population in an electronic state is distributed over a large number of rotational and vibrational levels $\left.\left(m=\mid \Lambda^{\prime \prime}, S^{\prime \prime}, \Sigma^{\prime \prime}, v^{\prime \prime}, J^{\prime \prime},(e / f)^{\prime \prime}\right)\right)$. The number of particles, $N_{m}$, in a specified level $E_{m}=E_{\Lambda^{\prime \prime}, S^{\prime \prime}, \Sigma^{\prime \prime}, v^{\prime \prime}, J^{\prime \prime},(e / f)^{\prime \prime}}$ is related to the total number of particles in all levels, $N$, according to the relation

$$
\frac{N_{m}}{N}=\frac{\left(2 J^{\prime \prime}+1\right) e^{-E_{m} / k T}}{Q_{\text {int }}}
$$

where $k$ is the Bolzmann constant, $T$ is the temperature, $\left(2 J^{\prime \prime}+1\right)$ is the degeneracy factor of the initial level, $e^{-E_{m} / k T}$ is the Boltzmann factor of the initial level, and $Q_{i n t}$ is the internal partition function. $Q_{\text {int }}$ is defined as the sum of the weighted Boltzmann factors of all discrete energy levels and can be expressed as (Tatum 1967)

$$
Q_{\text {int }}=Q_{e l} Q_{v i b} Q_{r o t}
$$

Here the partition function has been separated, just as the line strength in Eq. (4), into one electronic partition function, $Q_{e l}$, one vibrational partition function, $Q_{v i b}$, and one rotational partition function, $Q_{\text {rot }}$. The reader is referred to (Tatum 1967) for a detailed treatment of how to develop partition functions.

In order to calculate $Q_{\text {int }}$ one should sum over all states in a molecule (electronic, vibrational and rotational). This is impracticable and in practice one has to make some approximations. In particular it is not necessary, or even desirable, to extend the summation over all electronic states. Recipes for calculating partition functions have been published by Tatum (1966) and Sauval \& Tatum (1984). In order to assess how complete a calculation of an absorption coefficient over an extended wavelength region is, it is valuable to compare how large part of the partition function that is recovered. This can be done by summation over all energy levels included in the calculation of the absorption coefficient and by comparison with the partition function calculated according to the method described by Sauval \& Tatum (1984) (see also Jørgensen \& Larsson 1990). 


\section{Molecular opacity data}

In order to be useful for astrophysical applications, molecular absorption coefficients must be calculated for a large number of lines including very weak ones. Such calculations are performed on the basis of line lists containing $g f$-values for molecular lines. Molecular line lists have been developed by several groups based on essentially the methods outlined in the earlier sections. However, the applicability of a line list depends of course critically on the accuracy and completeness of the the underlying molecular data, and to what extent various approximations have been used to simplify the calculation of $g f$-values. It is not the intention of the present article to review and assess the various line data lists that are available for molecular astrophysics. The extensive work by Kurucz has been reviewed recently (Kurucz 1991) and is also described in another chapter of this book. Line lists for vibrational transitions within the ground electronic states of SiO, CO, CS and SiS have been developed by Tipping \& Chackerian (1981), Chackerian \& Tipping (1983) and Pineiro et al. (1987a, 1987b). Electronic transitions in CN, $\mathrm{C}_{2}$ and CO have been treated by Querci et al. (1974). The reader is referred to (Jørgensen 1992) for a more complete review of the subject.

The line data list developed (named SCAN-CN) for the CN "red" system by Jørgensen and the present author (Jørgensen \& Larsson 1990) has already been mentioned in the earlier sections. The SCAN-CN data base was developed exactly as outlined in Section 3. It contains $1.1 \times 10^{6}$ lines for each isotope combination ${ }^{12} \mathrm{C}^{14} \mathrm{~N},{ }^{12} \mathrm{C}^{15} \mathrm{~N},{ }^{13} \mathrm{C}^{14} \mathrm{~N}$ and ${ }^{13} \mathrm{C}^{15} \mathrm{~N}$. It carries, for each rotational line of the $\mathrm{A}^{2} \Pi-\mathrm{X}^{2} \Sigma^{+}$transition, the vibrational quantum number of the $\mathrm{A}^{2} \Pi$ and $\mathrm{X}^{2} \Sigma^{+}$states, the rotational quantum number of the $\mathrm{X}^{2} \Sigma^{+}$state (more specifically $J^{\prime \prime}+0.5$ ), the type of branch (see Fig. 2), the direction of the transition ${ }^{4}$, the $g f$-value $\left(=\left(2 J^{\prime \prime}+1\right) f_{v^{\prime} J^{\prime}, v^{\prime \prime} J^{\prime \prime}}\right)$, the vacuum line wavenumber $\left(\right.$ in $\left.\mathrm{cm}^{-1}\right)$ and the excitation energy (in $\left.\mathrm{cm}^{-1}\right)$ above $\mathrm{X}^{2} \Sigma^{+}\left(v^{\prime \prime}=0, J=0.5, F_{1}\right)$. Some comments should be made regarding the line wavenumbers. The wavenumber of a particular rotational line was obtained by subtracting the energy of the lower level, $E_{\Lambda^{\prime \prime}, S^{\prime \prime} \Sigma^{\prime \prime}, v^{\prime \prime}, J^{\prime \prime},(e / f)^{\prime \prime}}$, from the energy of the upper level, $E_{\Lambda^{\prime}, S^{\prime} \Sigma^{\prime}, v^{\prime}, J^{\prime},(e / f)^{\prime}}$. The energy levels were calculated by applying analytical expressions describing the energy level structure of the $\mathrm{A}^{2} \Pi$ and $\mathrm{X}^{2} \Sigma^{+}$states. This is nowadays not the optimal way to calculate energy levels in molecules and not the optimal use of molecular parameters derived from spectroscopy (see Lefebvre-Brion \& Field 1986), but it served the practical purpose of generating a large number of lines in a computationally simple way. When SCAN-CN is used for opacity calculations, the accuracy of individual line positions is of limited concern. Owing to the identification system in the data base it can also conveniently be used to generate synthetic spectra. The deviation between calculated and measured line position was checked for the $(6,0)$ and $(0,0)$ bands (Jørgensen \& Larsson 1990) and was found to be typically $0.2 \mathrm{~cm}^{-1}$ and $0.07 \mathrm{~cm}^{-1}$, respectively. No systematic check

${ }^{4}$ For a low lying state like $\mathrm{A}^{2} \Pi$ there is always the possibility for a transition $\mathrm{X}^{2} \Sigma^{+} \leftarrow \mathrm{A}^{2} \Pi$, where the arrow $\leftarrow$ is used to indicate that the $\mathrm{A}^{2} \Pi$ state is the lower state in the absorption process. 
against all measured lines were performed and there may be lines which deviate more than $0.2 \mathrm{~cm}^{-1}$. If SCAN-CN is used for the purpose of spectral analysis one should be aware of these limitations and exercise the necessary caution.

We will end this section with a brief discussion of the radiative properties of the $\mathrm{A}^{2} \Pi-\mathrm{X}^{2} \Sigma^{+}$transition of $\mathrm{CN}$. This discussion will serve to illustrate the difficulties one sometimes have to face in order to assess the accuracy of molecular data, be it experimental or theoretical. When the first planning of the SCAN-CN line list began, it seemed like the radiative properties of the CN "red" system was fully understood. Lifetime measurements of the six lowest vibrational levels of the $A^{2} \Pi$ state (Taherian \& Slanger 1984) were in very good agreement with results from $a b$ initio calculations (Larsson et al. 1983). However, more recent $a b$ initio calculations using a slightly different approach gave lifetimes about 30\% longer (Bauschlicher et al. 1988, Knowles et al. 1988). In terms of computational efforts these calculations are the most extensive so far for the CN "red" system. Furthermore, measurements of oscillator strengths for the $\mathrm{A}^{2} \Pi-\mathrm{X}^{2} \Sigma^{+}$transition (Davis et al. 1986) supported the more recent ab initio work. At this point it seemed like there was sufficient evidence for a modification of the $g f$-values in the SCAN-CN tape, and in (Jørgensen \& Larsson 1990) it is in fact suggested that the $g f$-values should be multiplied by a factor 0.734 in order to agree with the electronic transition moment function calculated by Bauschlicher et al. (1988) and Knowles et al. (1988). But the situation has once again changed. Lu et al. (1992) have measured radiative lifetimes by means of the laser induced fluorescence technique and found lifetimes even shorter than those predicted by Larsson et al. (1983). Thus it seems like the the CN "red" system is bound to remain an enigma for yet some time to come; after the experimental efforts by Lu et al. (1992) it is once again time for theoretical work in order to shed some new light on the problem.

\section{Final remarks}

It has been the intention of this review to provide a guidance for astrophysicists in need of data for modelling the absorption of radiation by molecules in the stellar environment. Much of the material can be found elsewhere, however, one would need to turn to several different sources in order to find it. At a first glance it would seem like a diatomic molecule, at least compared to a complex stellar environment, would constitute a rather simple system. However, diatomic molecules present many chances for errors, and they are very demanding systems for both experimentalists and theorists. This, among other things, is what makes them fascinating systems for studies in their own right.

Acknowledgements. The author would like to thank J.R. Mowat and L. Veseth for valuable comments on the manuscript. 


\section{References}

Bauschlicher C.W., Langhoff S.R., 1987, J. Chem. Phys., 87, 4665

Bauschlicher C.W., Langhoff S.R., Taylor P.R., 1988, Astrophys. J., 332, 531

Chackerian Jr. C., Tipping R.H., 1983, J. Mol. Spectrosc., 99, 431

Condon U., Shortley G.H., 1935, The Theory of Atomic Spectra, Cambridge Univ. Press, London

Davis S.P., Shortenhaus D., Stark G., Engleman Jr. R., Phillips J.G., Hubbard R.P., 1986, Astrophys. J., 303, 892, ibid. 307, 414

Hougen J.T., 1970, Natl. Bur. Std. Monogr., 115

Jarmain W.R., 1971, J. Quant. Spectrosc. Radiat. Tranfer, 11, 421

Jarmain W.R., McCallum J.C., 1971, Program TRAPRB Handbook CRESS York University, Toronto, Ontario

Jørgensen U.G., 1992, Revista Mexicana de Astronomia y Astrofisica, 23, 49

Jørgensen U.G., Larsson M., 1990, Astron. Astrophys., 238, 424

Kurucz R.L., 1991, in Stellar Atmospheres, Beyond Classical Models, eds Crivellari et al., NATO ASI, Kluwer, Dordrecht

Kovacs I., 1969, Rotational Structure in the Spectra of Diatomic Molecules, Hilger, London

Knowles P.J., Werner H.-J., Hay P.J., Cartwright D.C., 1988, J. Chem. Phys., 89, 7334

Larsson M., 1983, Astron. Astrophys., 128, 291

Larsson M., Siegbahn P.E.M., 1983, J. Chem. Phys., 79, 2270

Larsson M., Siegbahn P.E.M., Ågren, H.A., 1983, Astrophys. J., 272, 369

Lefebvre-Brion H., Field R.W., 1986, Perturbations in the Spectra of Diatomic Molecules, Academic, Florida

Lu R., Huang Y., Halpern J.B., 1992, Astrophys. J., 395, 710

Pineiro A.L., Tipping R.H., Chackerian Jr. C., 1987a, J. Mol. Spectrosc., 125, 91

Pineiro A.L., Tipping R.H., Chackerian Jr. C., 1987b, J. Mol. Spectrosc., 125, 184

Sauval A.J., Tatum J.B., 1984, Astrophys. J. Suppl., 56, 193

Schadee A., 1971, Astron. Astrophys., 14, 401

Taherian M.R., Slanger T.G., 1984, J. Chem. Phys., 81, 3814

Tatum J.B., 1966, Publ. Dominion Astrophys. Obs., 13, No. 1

Tatum J.B., 1967, Astrophys. J. Suppl., 14, 21

Tipping R.H., Chackerian C.Jr., 1981, J. Mol. Spectrosc., 88, 352

Veseth L., 1986, Symmetries and Rotational Line Intensities in Diatornic Molecules, Report 86-05, Institute of Physics, University of Oslo, Oslo

Whiting E.E., 1972, Ph.D. Thesis, York University, Toronto, Ontario

Whiting E.E., 1973, Computer Program for Determining Rotational Line Intensity Factors for Diatomic Molecules, NASA TN D-7268

Whiting E.E., Nicholls R.W., 1974, Astrophys. J. Supp., 27, 1

Whiting E.E., Schadee A., Tatum J.B., Hougen J.T., Nicholls R.W., 1980, J. Mol. Spec., 80, 249 